\title{
MITTEILUNGEN DER GESELLSCHAFT FÜR INFORMATIK / 230. FOLGE
}

Gesellschaft für Informatik e.V. (GI), Wissenschaftszentrum, Ahrstraße 45, 53175 Bonn, Tel.: 0228/302-145, Fax 0228/302-167, e-mail: gs@gi.de, Server: http://www.gi.de

Geschäftsfuhrung: Dr. Peter Federer, e-mail: peter.federer@gi.de, Tel.: -145

Stellvertreterin der Geschaftsführung: Cornelia Winter, e-mail: cornelia.winter@gi.de, Tel.: -147

Stellvertreter des Geschäftsführers: Alexander Rabe, e-mail: alexander.rabe@gi.de

Sekretariat: Monika Schulte, e-mail: monika.schulte@gi.de, Tel.: - 145

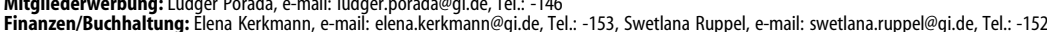

Mitgliederverwaltung: Tanja Degegeler, e-mail: tanja.diegeler@gi.de, Tel.. - 149 , Karola Schmitt, e-mail: karola.schmitt@gi.de, Tel.. - -151

Mitgliederverwaltung: Tanja Diegeler, e-mail: tanja.diegeler@gi.de,
ITK: Christopher Schiller, e-mail: christopher.schiller@gi.de, Tel.:-156

\section{Grand Challenges der Informatik}

Vor welchen großen Herausforderungen steht die Informatik in den kommenden Jahren und Jahrzehnten? Mit dieser Frage hat sich die Gesellschaft für Informatik e. V. (GI) im Rahmen der Initiative „Grand Challenges der Informatik“ beschäftigt. Gesucht haben wir grundsätzliche und schwierige Fragestellungen, deren Lösung mithilfe der Informatik einen deutlich spürbaren Fortschritt in wirtschaftlicher, sozialer oder gesellschaftlicher Hinsicht für die Allgemeinheit bedeutet. Fünf Grand Challenges haben wir schließlich aus den Vorschlägen destilliert, die wir in einer Broschüre zusammengefasst haben und als Poster veröffentlichen werden. In diesem Heft finden Sie nun das erste Poster unserer neuen Reihe „Grand Challenges der Informatik“, die nächsten vier legen wir den folgenden Heften bei.

Die GI hat diese fünf Fragestellungen als große Herausforderungen der Informatik identifiziert, weil sie damit zum Ausdruck bringen möchte, dass die Informatik sich zuallererst um die von der Informatik selbst geschaffenen Probleme kümmern muss:

- Wie bewahren wir digitale Informationen für unsere Nachwelt auf? Bücher, Bilder und Tonträger lassen sich ins Museum stellen. Sie lassen sich sogar digitalisieren und digi- talisiert für lange Zeit archivieren. Wie aber bewahrt man einen VideoClip, der digital erstellt und nur im Internet veröffentlicht wird, für die nachfolgenden Generationen auf?

- Wie erkennen wir beim Versenden einer digitalen Information, ob sie auf dem Weg zum Empfänger unbemerkt gelesen wurde? An einem versiegelten Briefumschlag sah man, ob das Siegel gebrochen war. Eine digitale Information kann unbemerkt kopiert, aber auch sehr einfach manipuliert worden sein. Wie sichern wir dann Integrität und Vertraulichkeit im Netz?

- Eine digitale Information kann mit beliebig vielen anderen Informationen verbunden werden. Es entstehen dabei vernetzte Systeme, die in ihrer Komplexität kaum fassbar, geschweige denn beherrschbar sind. Wie schaffen wir es, die daraus entstehenden Risiken einzudämmen und $\mathrm{zu}$ beherrschen?

- Ist es schon schwer, sich in einem Irrgarten zurechtzufinden, wo Wege im Nichts enden, wie schwer finden sich Menschen im virtuellen Raum zurecht? Wie kann die Mensch-Computer-Interaktion künftig so gestaltet werden, dass alle Bürger sich in der gemischt digitalphysikalischen Welt souverän bewegen können?

- Und nicht zuletzt: Wenn Software unsere Welt regiert, unsere Autos und Flugzeuge steuert und unsere medizinischen Instrumente dirigiert, wie schaffen wir es, zu beweisen, dass die Software genau das tut, was sie soll?

Die Auswahl spiegelt sowohl die Kompetenz der Informatik zur Definition

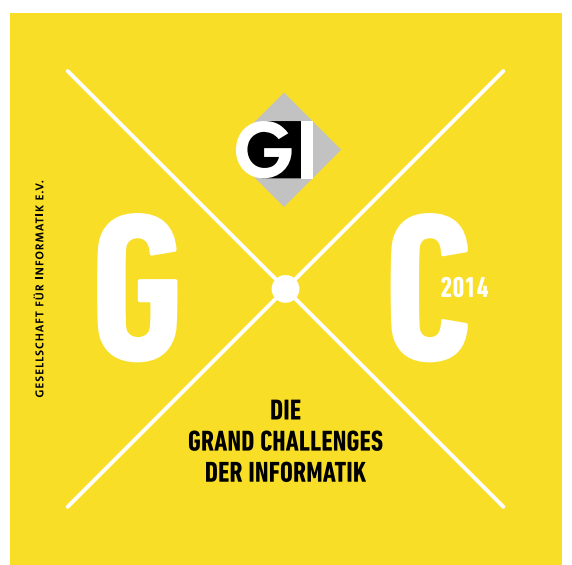

dieser Fragestellungen als auch ihre Verantwortung für die Gestaltung der Zukunft wider. Es werden Forschungsund Entwicklungsthemen benannt, die in der Fortentwicklung der Informatik als Schlüsselfelder gelten und Potenziale für künftige Anwendungen, Dienste oder Produkte beinhalten. Die Grand Challenges-Initiative soll Aufmerksamkeit bei Fachleuten und Interessenten in Wissenschaft, Politik, Gesellschaft schaffen, Ressourcen zur Lösungsfindung bündeln und den Nachwuchs motivieren.

Weitere Informationen und die Grand Challenges im Volltext finden Sie unter http://www.gi.de/themen/ grand-challenges-der-informatik.html.

\section{Simone Rehm \& Cornelia Winter}

\section{GI-Jahresbericht im Web}

Den GI-Jahresbericht 2013/2014 finden Sie im Internet unter www.gi. de/service/downloads. Bei Bedarf schicken wir ihn Ihnen gerne auch in gedruckter Form zu: gs@gi.de.

DOI 10.1007/s00287-014-0842-0

Die Fortsetzung der Mitteilungen folgt auf Seite 614. 